\title{
ANALOGICAL REINFORCEMENT LEARNING
}

by

\section{JAMES MICHAEL FOSTER}

B.S., University of Florida, 2009

A thesis submitted to the

Faculty of the Graduate School of the

University of Colorado in partial fulfillment

of the requirements for the degree of

Master of Arts

Department of Psychology and Neuroscience

2015 
This thesis entitled:

ANALOGICAL REINFORCEMENT LEARNING written by JAMES MICHAEL FOSTER

has been approved for the Department of Psychology and Neuroscience

Matt Jones

Albert Kim

Date

The final copy of this thesis has been examined by the signatories, and we find that both the content and the form meet acceptable presentation standards of scholarly work in the above mentioned discipline.

IRB protocol \#11-0018 \& \#14-0609 
FOSTER, JAMES MICHAEL (M.A., Cognitive Psychology)

\section{ANALOGICAL REINFORCEMENT LEARNING}

Thesis directed by Matt Jones

How do people learn new abstract concepts? The approach taken in this work is to develop a theoretical and computational framework for how such concepts are learned and applied. The framework integrates established principles of cognition (analogy and reinforcement learning) and explores their computational power and empirical validity. The first section of this thesis presents computational models and simulation results in the domain of tic-tactoe. The goal of the computational model is to demonstrate a how a synthesis of established principles of cognition provides a framework for constructing abstract relational concepts and evaluating their usefulness. The second section describes experiments with humans that qualitatively test some of the model predictions. The goal of the experiments is to explore how feedback (in particular, reward) and frequency affect the reinforcement of relational concepts. The final section discusses implications of the modeling and empirical results and situates this work within relevant prior research. 


\section{Contents}

\section{Chapter}

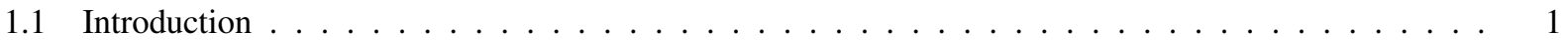

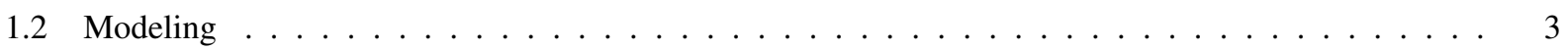

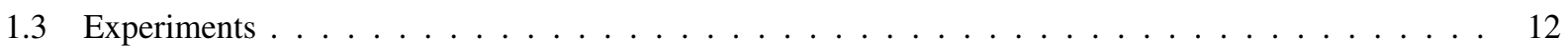

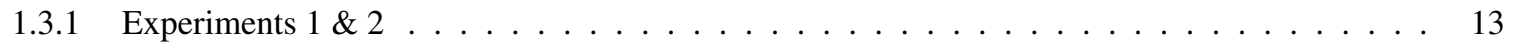

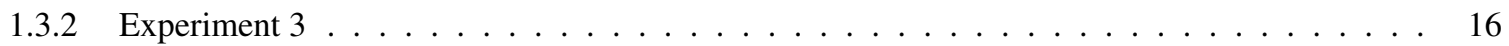

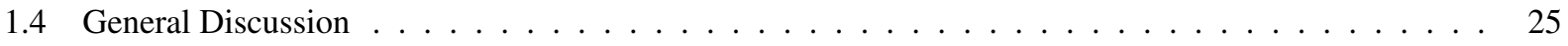

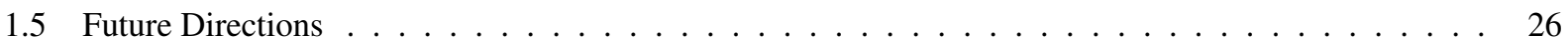

1.5.1 The Role of Language in Relational Concept Learning . . . . . . . . . . . . . . . . . . . 27

$\begin{array}{ll}\text { Bibliography } & 30\end{array}$ 


\section{List of Tables}

\section{Table}

1.1 Experiment 3 Conditions and Point Values. In the "A High Variance" condition (3rd column), category A had a half-width of 10 and category $\mathrm{C}$ had a half-width of 1 . In the "A Low Variance" condition (4th column), category A had a half-width of 1 and category $\mathrm{C}$ had a half-width of 10 . The means and half-widths of categories B and D were the same across conditions. Points were sampled from triangular distributions with these means and half-widths, and then were multiplied by a scaling factor

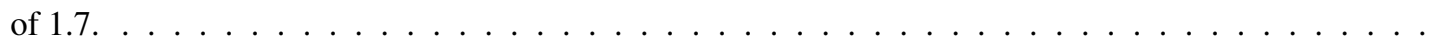




\section{List of Figures}

\section{Figure}

1.1 An example of structural alignment between two chess positions. Both positions contain instances of the abstract concept of a FORK: black's piece is simultaneously attacking both of white's pieces. These attacking relations are represented by the red arrows. Cyan lines indicate the mapping between the two episodes. The mapping satisfies parallel connectivity because it respects the bindings between

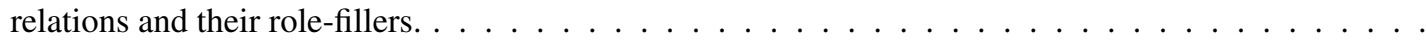

1.2 Schema Induction Model. Model operation. Each candidate afterstate is evaluated by analogical comparison to stored exemplars, followed by similarity-weighted averaging among the learned exemplar values. Learning is by TD error applied to the exemplar values. On some trials, especially useful analogies produce new schemas that are added to the exemplar pool. In the example here, $s$ and $E$ both have guaranteed wins for $\mathrm{X}$ by threatening a win in two ways. The induced schema embodies this abstract structure. Dots with red arrows indicate ternary "same-rank" relations. . . . . . . . . .

1.3 Simulation Results. The relational model learns faster than the featural model, and the schema induction model learns faster than the relational model. Performance is measured by points, which is the number of moves made before the game is a sure loss against an ideal player. . . . . . . . . . . .

1.4 State and Schema Attentions and Values for one copy of the Schema Induction model at the end of 50,000 training games. A. Schemas tend to have more extreme values (v) than states, which indicates they are more diagnostic of winning and losing. B. Schemas tend to have larger attentions (u) than states, which indicates they are more useful. 
1.5 Example of the top representations (based on most extreme values of attention $u$ ) for one copy of the Schema Induction model after 50,000 training games. . . . . . . . . . . . . . . . . . . . . 10

1.6 Unguided Baselines. For these baseline simulations, schemas were induced randomly, with the number and size of schemas induced yoked to guided schema induction model. . . . . . . . . . . . .

1.7 Training Trial Stimuli Examples. Left: Category A stimulus presented during a training trial. Right: Category B stimulus presented during a training trial. . . . . . . . . . . . . . . 14

1.8 Test Trial Stimuli Examples. Left: Unambiguous Test Trial. Right: Ambiguous Test Trial. $\ldots \ldots \ldots$

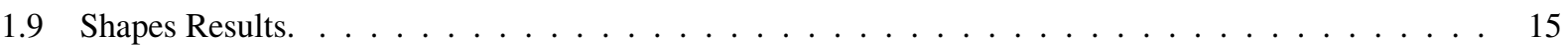

1.10 Stimuli Examples for Experiment 3. . . . . . . . . . . . . . . . . . . . . . . . . . . . . . 19

1.11 Predictions for Experiment 3. The horizontal lines represent abstract number lines and the A, B, and $\mathrm{C}$ triangles represent the means and variances of their respective triangular distributions. We predicted that the valuation of the $\mathrm{AC}$ items would be shifted from the arithmetic mean of $\mathrm{A}$ and $\mathrm{C}$ (which was equal to the mean of B) towards the lower-variance category. In the "A is High Variance" condition (top, in red), it was predicted that the valuation of $\mathrm{AC}$ items would be shifted towards $\mathrm{C}$, resulting in participants selecting B over AC in critical test trials. In the "A is Low Variance" condition, it was predicted that the valuation of $\mathrm{AC}$ items would be shifted towards $\mathrm{A}$, resulting in participants selecting

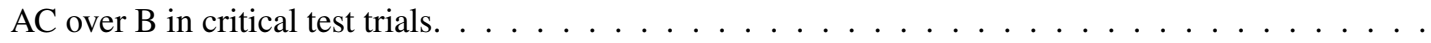

1.12 Critical Test Trials. Condition significantly predicts choice of AC in AC vs. B trials in the expected direction $($ difference in means $=2.28, p<.05$ by both t-test and Mann-Whitney) $\ldots \ldots \ldots$

1.13 Training Trial Accuracy. There was a significant interaction between Trial Type and Condition in accuracy on training trials $(t(78)=-2.07, p<.05$ by mixed model analysis of variance $)$. Accuracy is better on $\mathrm{AB}$ trials when $\mathrm{A}$ is low variance than when $\mathrm{A}$ is high variance (left). Accuracy is better on $\mathrm{BC}$ trials when $\mathrm{C}$ is low variance than when $\mathrm{C}$ is high variance (right). For this figure, blocks were defined by averaging over sequences of 20 trials. Participants who reached criterion were assigned an accuracy of 1 for trials they were not presented. 
1.14 Test Trial Response Times. There was a significant interaction between Trial Type (AB v. BC) and Condition on response time $(t(88)=2.00, p<.05)$. Response time is faster on $\mathrm{AB}$ trials when $\mathrm{A}$ is low variance than when $\mathrm{A}$ is high variance, and faster on $\mathrm{BC}$ trials when $\mathrm{C}$ is low variance than when

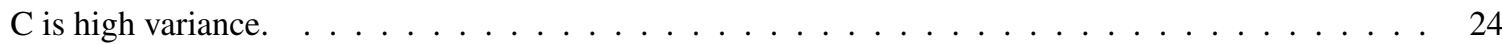




\section{$1.1 \quad$ Introduction}

How do people learn new abstract concepts? Everyone has a repertoire of concepts they use in making sense of the world and filtering the infinite complexity of experience into manageable chunks. Abstract representations that capture reoccurring patterns in the environment are used for generalization (i.e., making predictions or inferences about states of the environment that haven't been experienced before). How are these representations constructed, and how is their usefulness evaluated? For example, how do people discover and apply concepts such as a 'fork' in the game of chess? How do scientists create theories such as natural selection?

The approach taken in this work is to develop a theoretical and computational framework for how such concepts are learned and applied. The framework integrates established principles of cognition and explores their computational power and empirical validity. The principles integrated here are analogy and reinforcement learning (RL).

Analogy is a powerful cognitive tool for abstract reasoning, far transfer, and creativity. Analogical learning involves discovering relational structure that is shared by two situations (Gentner, 1983). Schema induction is the process of creating a new abstract concept that's derived from an analogy (Doumas et al., 2008; Hummel and Holyoak, 2003). The induced schema embodies structure common to both analogues. However, schema induction needs guidance to select the patterns most useful for a given task.

Reinforcement Learning (RL) is a powerful framework for trial and error learning that works by estimating the values of states and actions (Sutton and Barto, 1998). However, in order to learn efficiently, RL needs a sophisticated similarity function for generalizing values across states.

There is a complementary relationship between analogy and RL, which hints at the potential for a computationally powerful, synergistic interaction between these two cognitive processes. The first proposed connection between analogy and RL is that structural alignment yields an abstract form of psychological similarity that can support sophisticated generalization (Gick and Holyoak, 1980; Markman and Gentner, 1993). Incorporating analogical similarity into the RL framework could thus lead to rapid learning in complex, structured environments. For example, an RL model of chess equipped with analogical similarity should recognize the similarity between the two positions in Figure 1.1 and hence generalize between them. Consequently the model should learn to create forks and to avoid forks by the opponent much more rapidly than if it had to learn about each possible fork instance individually. 


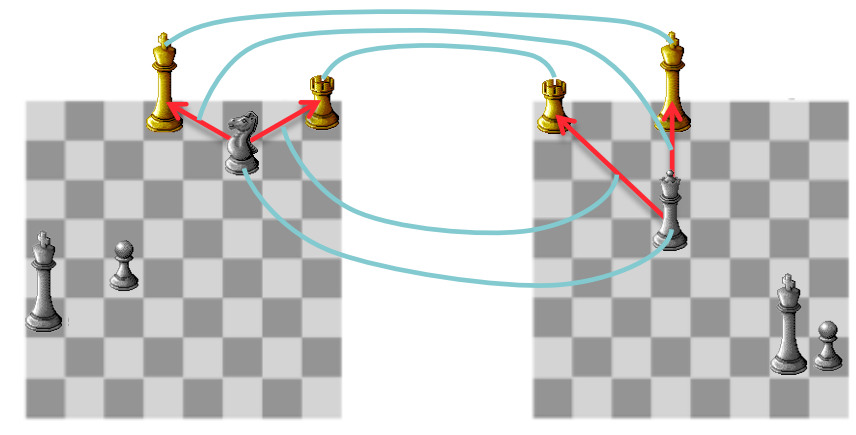

Figure 1.1: An example of structural alignment between two chess positions. Both positions contain instances of the abstract concept of a FORK: black's piece is simultaneously attacking both of white's pieces. These attacking relations are represented by the red arrows. Cyan lines indicate the mapping between the two episodes. The mapping satisfies parallel connectivity because it respects the bindings between relations and their role-fillers.

The second proposed connection is that the TD error computed by RL models, for updating value estimates, can potentially drive analogical learning by guiding schema induction and attention. Instead of forming schemas for whatever relational structures are frequently encountered (or are discovered by analogical comparison of any two states), an analogical RL model can be more selective, only inducing schemas from analogies that significantly improve reward prediction. Such analogies indicate that the structure common to the two analogue states may have particular predictive value in the current task, and hence that it might be worth extracting as a standalone concept. For example, if the model found a winning fork move by analogical comparison to a previously seen state involving a fork, the large boost in reward could trigger induction of a schema embodying the abstract concept of a fork. Furthermore, the model can use prediction error to increase attention to concepts that are more useful for predicting reward, and decrease attention to concepts that are less useful. Such an attention-learning mechanism is proposed to bias the model to rely more on concepts that have been consistently useful in the past.

Analogical RL thus integrates three principles from prior research: RL, exemplar generalization, and structural alignment of relational representations. Because each of these principles has strong empirical support as a psychological mechanism, it is plausible that they all interact in a manner similar to what is proposed here. Thus it seems fruitful to explore what these mechanisms can achieve when combined.

The first section of this thesis presents computational models and simulation results in the domain of tic-tactoe. The goal of the computational model is to demonstrate a how a synthesis of established principles of cognition (analogy and reinforcement learning) provides a framework for constructing abstract relational concepts and evaluating 
their usefulness. The second section describes experiments with humans that qualitatively tests some of the model predictions. The goal of the experiments is to explore how feedback (in particular, reward) and frequency affect the reinforcement of relational concepts. The final section discusses implications of the modeling and empirical results and situates this work within relevant prior research.

\subsection{Modeling}

The goal of the model is to develop a computational understanding of how people learn abstract concepts. Research in analogical reasoning suggests that higher-order cognitive functions such as abstract reasoning, far transfer, and creativity are founded on recognizing structural similarities among relational systems. However, a critical element is missing from these theories, in that their operation is essentially unsupervised, merely seeking patterns that recur in the environment, rather than focusing on the ones that are predictive of reward or other important outcomes. The model integrates theories of analogy with the computational framework of reinforcement learning (RL). We propose a computational synergy between analogy and RL, in which analogical comparison provides the RL learning algorithm with a measure of relational similarity, and RL provides feedback signals that can drive analogical learning. We formalized this integration in a model that learns to play tic-tac-toe. The model uses RL to incrementally learn value estimates of stored exemplars and schemas. These estimates are used to predict win probabilities for different game states by similarity-weighted averaging, where similarity is determined by the quality of analogical mappings. On some trials, especially useful analogies produce new schemas that are added to the pool. Simulation results support the power of this approach.

\subsubsection{Model}

The proposed model works as follows (see equations in Figure 1.2). The model maintains a set of exemplars $E$, each with a learned value, $v(E)$. To estimate the value of any state $s$, it compares that state to all exemplars by structural alignment, which yields a measure of analogical similarity for each exemplar (Forbus and Gentner, 1989).

The estimated value of the state, $\tilde{V}(s)$, is then obtained as a similarity-weighted average of $v(E)$. After any action is taken and the immediate reward and next state are observed, a TD error is computed as in standard RL. The exemplar values are then updated in proportion to the TD error and in proportion to how much each contributed to the model's 
prediction, that is, in proportion to $\operatorname{sim}(s, E)$. The attentions $u(E)$ implement exemplar-specific attentions weights or learning rates. The $u(E)$ values can also be thought of as voting weights in the computation of $\tilde{V}(s)$. Exemplars with higher $u$ values have greater influence on the similarity-weighted average of exemplar values. The attentions are also updated in proportion to the TD error, in proportion to how much each contributed to the model's prediction, and in proportion to how much that exemplar's prediction differed from the overall prediction $\tilde{V}$. Attention is increased to exemplars that individually made a more accurate prediction that the overall prediction, and attention is decreased to exemplars that individually made a less accurate prediction than the overall prediction.

Additionally, whenever the structural alignment between a state and an exemplar produces a sufficient reduction in prediction error (relative to what would be expected if that exemplar were absent), a schema is induced from that analogy. The schema is an abstract representation, defined on token (placeholder) objects, and it contains only the shared information that was successfully mapped by the analogy. The schema is added to the pool of exemplars, where it can acquire value associations directly (just like the exemplars do). The advantage conferred by the new schema is that it allows for even faster learning about all states it applies to (i.e., that contain that substructure). For example, rather than learning by generalization among different instances of forks, the model would learn a direct value for the fork concept, which it could immediately apply to any future instances. A consequence of the schema induction mechanism is that the pool of concrete exemplars comes to contain more and more abstract schemas. Thus the model's representation transitions from initially episodic to more abstract and conceptual.

The simulation study presented below uses a variant of RL known as afterstate learning, in which the agent learns values for the possible states it can move into (Sutton and Barto, 1998). This is a reasonable and efficient method for the task we use here-tic-tac-toe, or noughts \& crosses—because the agent's opponent can be treated as part of the environment and is the only source of randomness. Our main proposal regarding the interaction between RL and analogical learning is not limited to this approach.

The operation of the model is illustrated in Figure 1.2. On each time step, the model identifies all possible actions and their associated afterstates. For each afterstate $s$, it computes an analogical similarity, $K$, to each exemplar, $E$, by structural alignment. Each possible mapping $M: s \rightarrow E$ is evaluated according to

$$
\Phi(M)=\beta \cdot \sum_{o \in s} \operatorname{sim}(o, M(o))+\sum_{r \in s} \operatorname{sim}(r, M(r)) \cdot\left[1+\sum_{i=1}^{n_{r}} I_{\left\{M\left(\operatorname{child}_{i}(r)\right)=\operatorname{child}_{i}(M(r))\right\}}\right]
$$


This expression takes into account object similarity, by comparing each object $o$ in $s$ to its image in $E$; relational similarity, by comparing each relation $r$ in $s$ to its image in $E$; and parallel connectivity, by having similarity between mutually mapped relations "trickle down" to add to the similarity of any mutually mapped role-fillers (Forbus and Gentner, 1989). The sim function is a primitive (object- and relation-level) similarity function, $\beta$ determines the relative contribution of object similarity, $n_{r}$ is the number of roles in relation $r$, child ${ }_{i}(r)$ is the object filling the $i^{\text {th }}$ role of $r$, and $I_{\{P\}}$ is an indicator function equal to 1 when proposition $P$ is true. Analogical similarity is then defined as the value of the best mapping (here the $\theta$ parameter determines specificity of generalization):

$$
\operatorname{sim}(s, E)=\exp \left(\theta \cdot \max _{M} \Phi(M)\right) .
$$

The activation $a(E)$ of each exemplar is determined by normalizing the analogical similarities, and the estimated value of $s, \tilde{V}(s)$, is computed as a similarity-weighted average of the exemplar values $v(E)$ (Figure 1.2). Thus the estimate is based on the learned values of the exemplars most similar to the candidate state.

Once values $\tilde{V}(s)$ have been estimated for all candidate afterstates, the model uses a softmax (Luce-choice or Gibbs-sampling rule) to select what state to move into (here $\tau$ is an exploration parameter):

$$
\operatorname{Pr}\left[s_{t}=s\right] \propto e^{\tilde{V}(s) / \tau}
$$

Learning based on the chosen afterstate $s_{t}$ follows the SARSA rule (Rummery and Niranjan, 1994), after the model chooses its action on the next time step. This produces a TD error, which is then used to update the exemplar values and exemplar attention or voting weights by gradient descent (see Equations for $\Delta v(E)$ and $\Delta u(E)$ in Figure 1.2). This exemplar-specific attention weight learning mechanism (u parameter in the figure equations) allows the model to learn which exemplars are most useful for reducing prediction error. Over time, the model learns to increase attention to exemplars which are predictive of reward outcome, and decrease attention to exemplars which are not predictive of outcome. This attention learning mechanism is tested empirically in Experiments 1, 2, and 3, described below. 


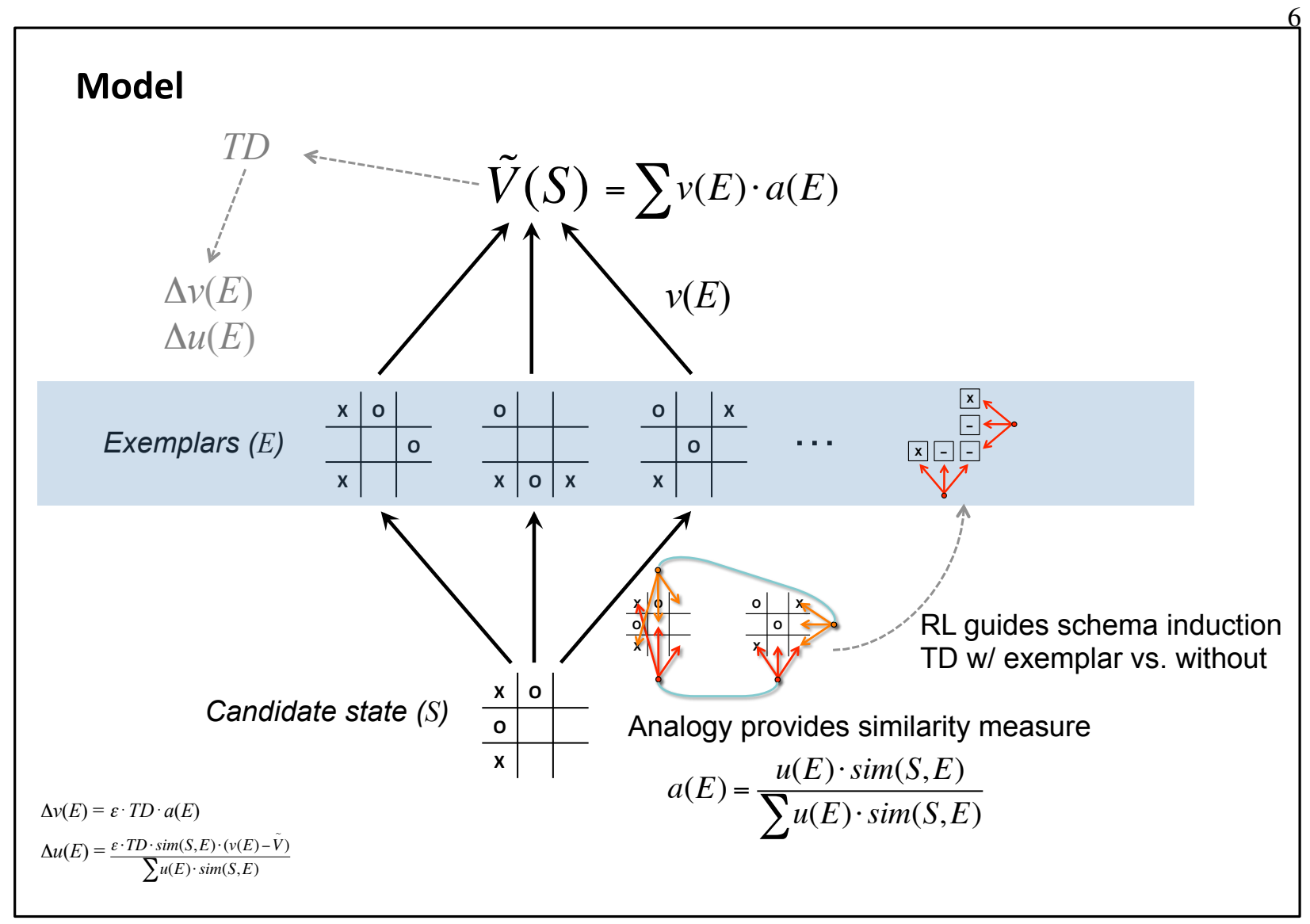

Figure 1.2: Schema Induction Model. Model operation. Each candidate afterstate is evaluated by analogical comparison to stored exemplars, followed by similarity-weighted averaging among the learned exemplar values. Learning is by TD error applied to the exemplar values. On some trials, especially useful analogies produce new schemas that are added to the exemplar pool. In the example here, $s$ and $E$ both have guaranteed wins for $\mathrm{X}$ by threatening a win in two ways. The induced schema embodies this abstract structure. Dots with red arrows indicate ternary "same-rank" relations.

\subsubsection{Simulation}

The analogical RL model was tested on its ability to learn tic-tac-toe (Foster and Jones, 2013). Each board position was represented by treating the nine squares as objects of types 0 (blank), 1 (focal agent's), and 2 (opponent's), and defining 8 ternary "same-rank" relations for the rows, columns, and diagonals. Thus a player wins by filling all squares in any one of these relations. Object similarity was defined as 1 for matching object types and 0 otherwise. Similarity between relations was always 1 because there was only one type of relation. Reward was given only at the 
end of a game, as +1 for the winner, -1 for the loser, or 0 for a draw. After the game ended, it moved to a special terminal state with fixed value of 0 . For simplicity, all free parameters of the model $(\beta, \theta, \alpha, \gamma, \tau)$ were set to a default value of 1 .

Three variations of the model were implemented, differing in their levels of analogical abstraction. The Featural model was restricted to literal mappings between states (upper-left square to upper-left square, etc.). This model still included generalization, but its similarity was restricted to the concrete similarity of standard feature-based models. The Relational model considered all 8 mappings defined by rigid rotation and reflection of the board. This scheme was used in place of searching all 9! possible mappings for every comparison, to reduce computation time. Finally, the Schema Induction model extended the Relational model by inducing schemas that capture the relational structure critical to the task. Following learning after each trial, the Schema Induction model determines how much each exemplar contributed to reducing prediction error, by comparing $T D$ to what it would have been without that exemplar. If the reduction is above some threshold, the analogical mapping found for that exemplar (lower right of figure 1.2) produces a schema that is added to the exemplar pool (far right). The schema is given a value of $v$ initialized at $\tilde{V}\left(s_{t}\right)$. This schema value is updated on future trials just as are the exemplar values. Acquisition of new schemas in this way is predicted to improve the model's pattern of generalization, tuning it to the most useful relational structures in a task.

\subsubsection{Results}

Each model variant was trained in blocks of 10 games of self-play followed by a pair of testing games against an ideal player (playing first in one game and second in the other). Learning occurred only during training. In testing games, the model was given one point for each non-losing move it made (i.e., moves from which it could still guarantee a draw), for a maximum of 9 points per pair of testing games. Average learning curves are shown in Figure 1.3 for eight independent copies of each model over 5000 blocks (50,000 training games). These results show that the Featural model does eventually learn, but the Relational model learns an order of magnitude faster, and the Schema Induction model learns even faster than the Relational model. 


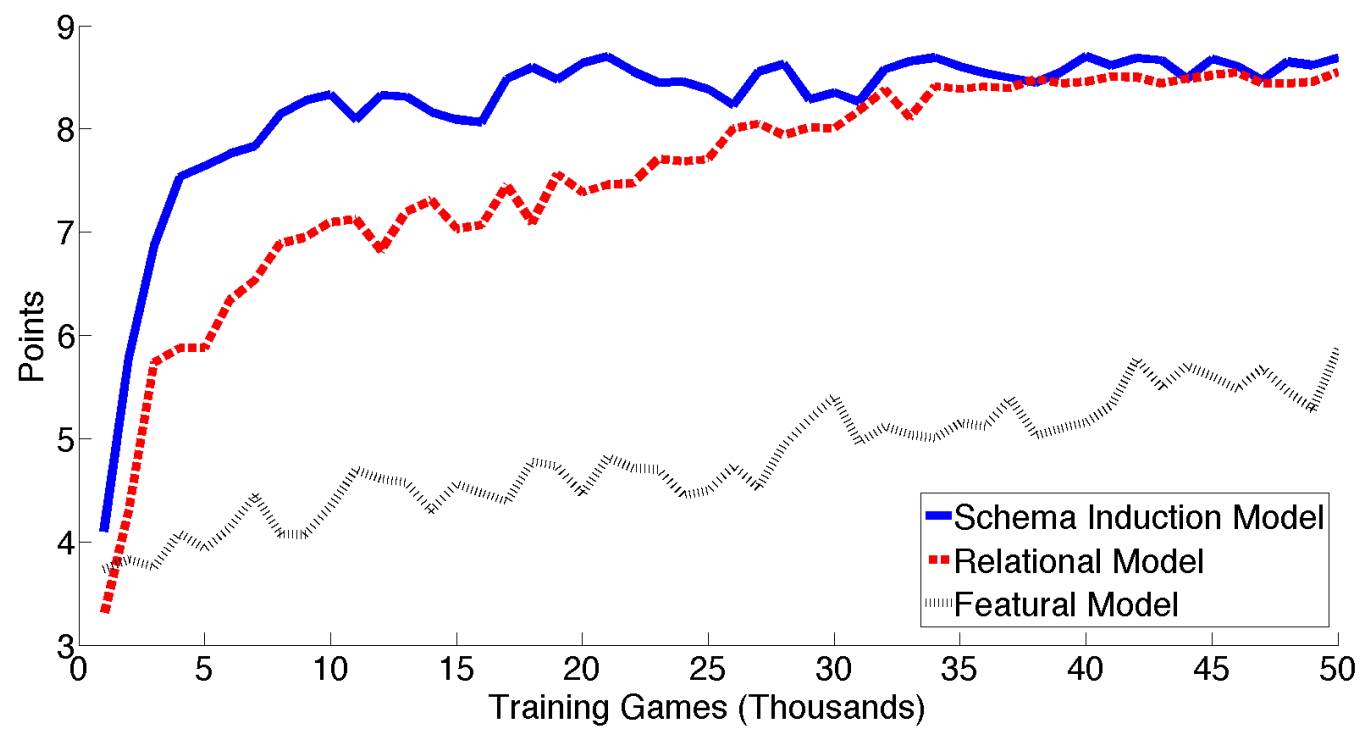

Figure 1.3: Simulation Results. The relational model learns faster than the featural model, and the schema induction model learns faster than the relational model. Performance is measured by points, which is the number of moves made before the game is a sure loss against an ideal player.

Figure 1.4 shows histograms of the learned values (v) and attentions (u) separately for states recruited as exemplars and schemas induced by the model and recruited as exemplars. The values for schemas (bottom left) tend to be more extreme (either highly positive or highly negative) than the values for states (top left). The bimodal value distribution for schemas suggests that schemas, overall, are more diagnostic of winning or losing board positions. Additionally, the attentions for schemas (bottom right) tend to be larger than attentions for states (top right). An exemplar with a higher attention value tends to make especially accurate reward predictions. The overall larger attentions for schemas (as compared to states) indicates that schemas are, overall, more useful exemplars for reward prediction. 

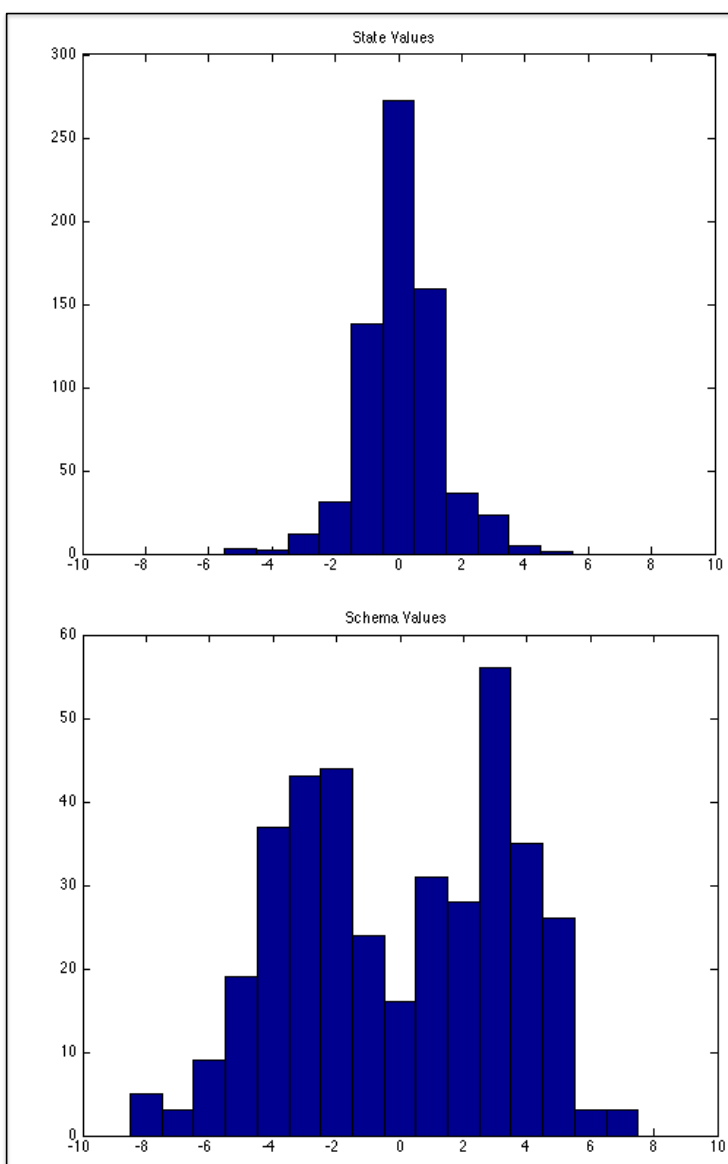

A
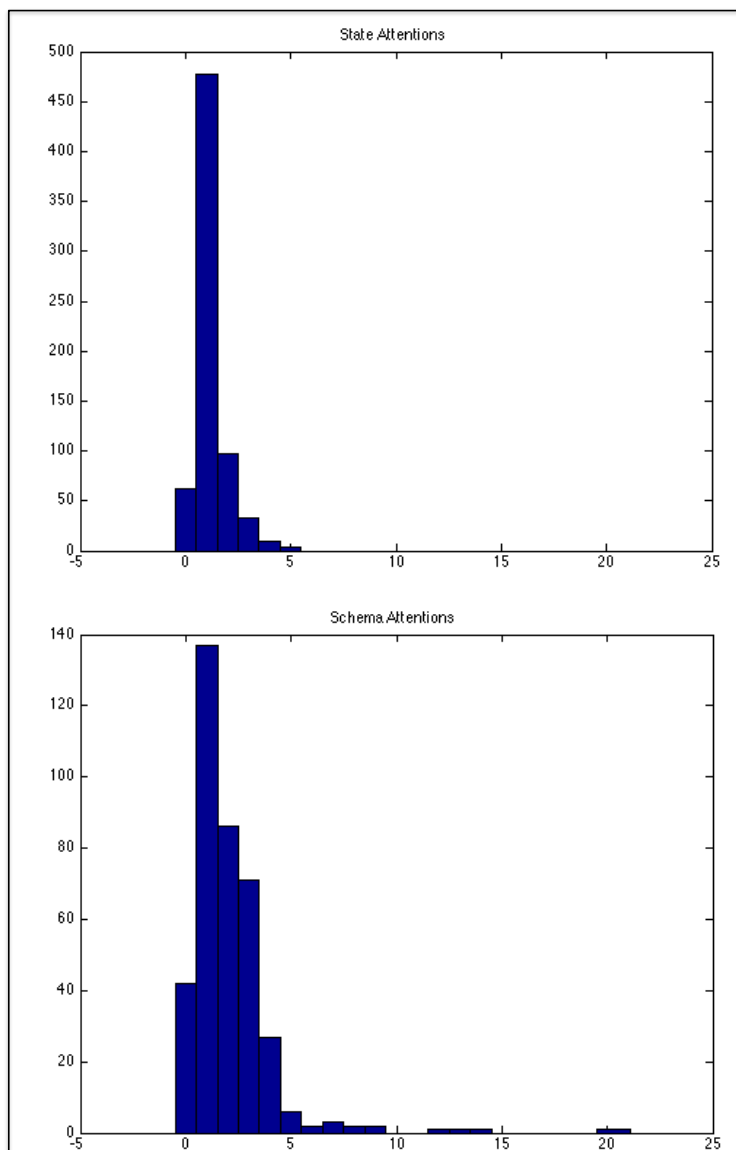

B

Figure 1.4: State and Schema Attentions and Values for one copy of the Schema Induction model at the end of 50,000 training games. A. Schemas tend to have more extreme values (v) than states, which indicates they are more diagnostic of winning and losing. B. Schemas tend to have larger attentions (u) than states, which indicates they are more useful.

Figure 1.5 shows examples of some of the most useful representations learned by the Schema Induction model. The top two and bottom left schemas represent winning states of tic-tac-toe. The top left schema perfectly matches (with a similarity of 1) any tic-tac-toe state with 3 O's in the left row, the right row, the top row, or the bottom row regardless of which objects (X, O, or blank) occupy the other grid locations. Similarly, the top right schema perfectly matches any tic-tac-toe state with 3 O's in the middle vertical or middle horizontal rows. Both the top left and top right schemas are "pure" or "clean" in that they contain no information that's irrelevant to the winning states. However, the bottom left schema does contain an irrelevant piece of information - the $\mathrm{X}$ in the bottom center grid location. This schema is "dirty" in that it contains irrelevant details from the states from which it was induced. With further training, 
the model would likely refine this schema and learn that abstracting out the X provides a more general, more useful schema. Finally, the bottom right schema represents the state in which the model is about to lose the game by an opponent making 3 X's in a diagonal row. This schema perfectly matches and tic-tac-toe state with 2 X's and in a row diagonally from top right to bottom left or from top left to bottom right and followed by a blank grid location. However, this schema would not be a perfect match to states of the form X-blank-X.

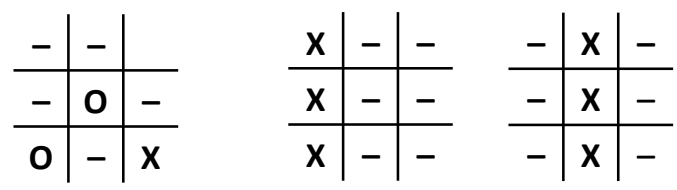

$$
\begin{aligned}
& \begin{array}{l|l|ll|l|ll|l|l}
- & - & 0 \\
\hline- & 0 & - \\
\hline & - & - & x & - & - & 0 & & - \\
\hline- & 0 & x & 0 & - & x
\end{array}
\end{aligned}
$$

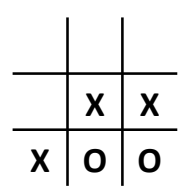

Figure 1.5: Example of the top representations (based on most extreme values of attention u) for one copy of the Schema Induction model after 50,000 training games.

\section{Unguided Schema Induction Baseline}

The computational model was extended to establish a new baseline. The new baseline included schema induction and relational similarity, but the schema induction process was not guided by RL feedback. Instead, this baseline model randomly induced schemas between exemplars and the current state. The number of schemas induced was determined by yoking to the feedback-guided schema induction model. Thus this yoked baseline model induced the same number of schemas as the feedback-guided schema induction model, but the particular schemas learned were induced from comparisons between the current state and randomly chosen exemplars. The hypothesis was that the feedback-guided schema induction model would learn faster than the yoked baseline model, and would also discover more useful representations. Figure 1.6 compares the guided schema induction model with learned attentions (Learned $\mathrm{U})$ to baselines with unguided induction and learned $\mathrm{u}$ (red) and unguided induction with fixed $\mathrm{u}$ (orange). There is a large improvement in performance when the model can learn to adapt its attentions based on prediction error. There is a further improvement when the model's schema induction process itself is also guided by reduction in prediction 
error.

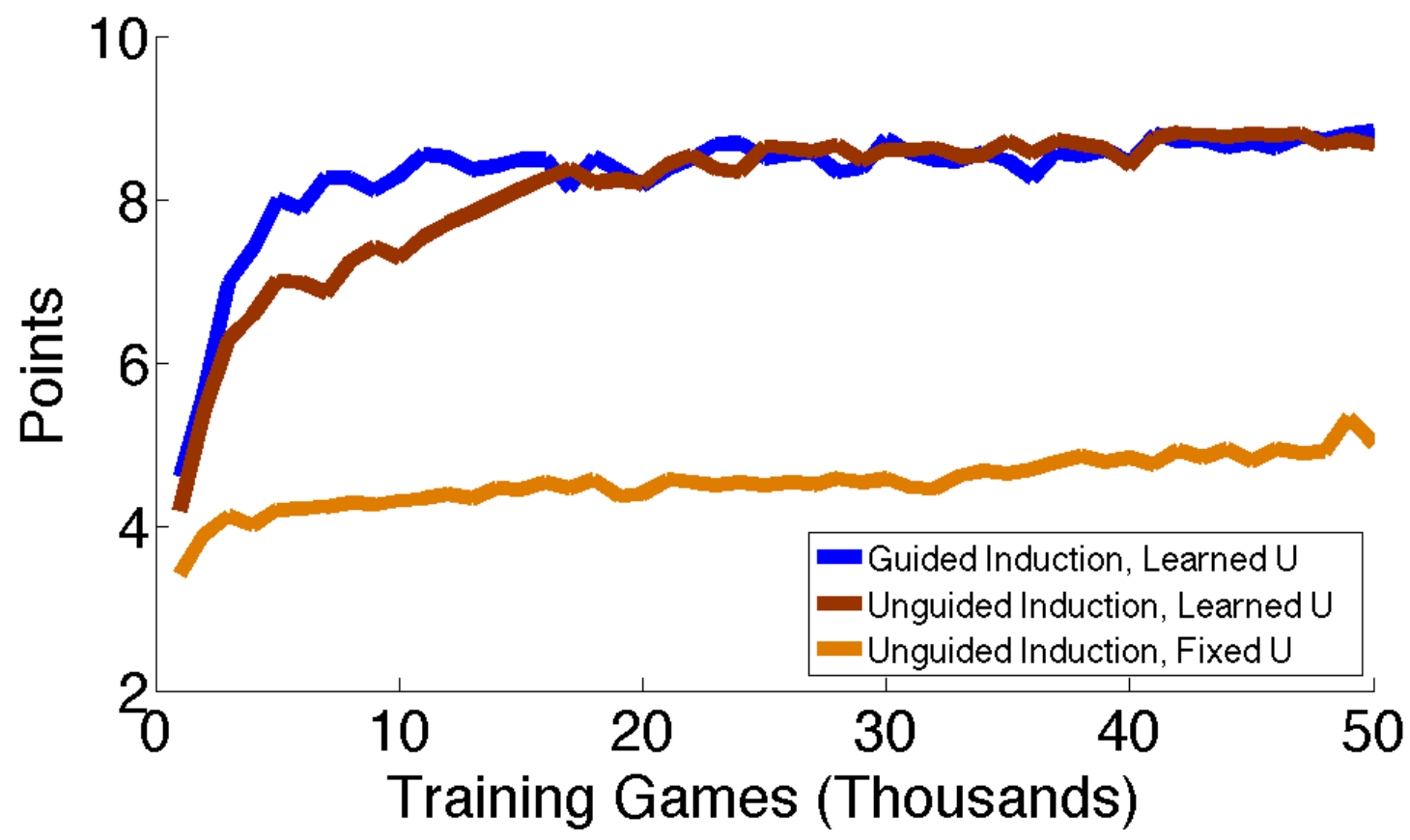

Figure 1.6: Unguided Baselines. For these baseline simulations, schemas were induced randomly, with the number and size of schemas induced yoked to guided schema induction model.

\subsubsection{Two-Stage Memory Retrieval}

Another extension to the computational model was to implement two-stage memory retrieval following the MAC/FAC model (Forbus et al., 1995). The first stage uses fast feature-vector similarity to efficiently retrieve a set of candidate exemplars, and the second stage uses structural alignment to determine the best analogical matches. Such two-stage retrieval enables a more computationally tractable and psychologically plausible form of analogical inference from stored exemplars and schemas to novel situations. This model also lays groundwork that will be useful for a later model with relational consolidation. In a model with consolidation, the exemplars that are learned to be most useful (via the attention-learning mechanism described above) would become new perceptual features which could be leveraged by the MAC retrieval stage to improve the candidate exemplars retrieved for the subsequent structural alignment processing.

The first stage of retrieval (MAC) computes a MAC score which is used to select the set of candidate exemplars 
that pass on to the second stage. The candidate exemplar set is defined by the top $\mathbf{N}$ exemplars sorted by descending MAC score. To compute each exemplar's MAC score, first the model computes the cosine similarity between each exemplar's feature vector and the candidate state's feature vector. The choice of cosine similarity is an implementational detail, and the model is not theoretically committed to this particular choice of featural similarity. This featural similarity measure is then multiplied by the exemplar-specific attention weights $\mathbf{u}$ to compute each exemplar's MAC score (see Equation 3). These two components (featural similarity and attention) in the MAC score make exemplars with higher featural similarity and higher attentions more likely to be retrieved from memory and included in the set of candidate exemplars. The relative weighting of the featural similarity and the exemplar attentions is set by an exponent p on the attention weight.

$$
M A C(S, E)=u(E)^{p} \frac{f(S) \cdot f(E)}{\|f(S)\|\|f(E)\|}
$$

A flat feature vector $\mathbf{f}$ was defined for each exemplar and candidate state of tic-tac-toe. The feature vector had dimensions for the number of X's, the number of O's, the number of blanks, and the number of unspecified squares (which indicates the size of schema). However, this feature vector did not produce good performance with a candidate exemplar set size of 10 or 100 . This poor performance may be due to lack of useful dimensions in the feature vector. With consolidation the model might learn new useful features that would improve the quality of the candidate exemplar set and improve performance. Future work will investigate these modeling issues in more detail.

\subsection{Experiments}

There are several possible approaches to investigating the mechanisms of abstract concept learning. One approach involves testing the model piecewise with qualitative demonstrations of the model's components (e.g., demonstrating that schemas can be associated with values, that generalization of schema values is proportional to relational similarity, or that reward experienced as a result of application of a schema makes that schema more likely to be retrieved and applied in the future). Another approach involves testing the model holistically with quantitative predictions about human task performance metrics such as response time or accuracy. These approaches are limited in the extent that specific components of the model can be tested, but enable fitting the model to human data. The 
experiments described below take the qualitative approach.

\subsubsection{Experiments $1 \& 2$}

The goal of Experiments $1 \& 2$ was to test the effects of many-trial schema reinforcement and frequency using visual stimuli. Participants learned to classify relational configurations of shapes into two categories that differed in frequency of instantiation or reward. Following training, participants completed partial category instances that were either unambiguous (could be correctly completed as only one category) or ambiguous (could be correctly completed as either category). We predict that category completion of ambiguous items will be biased toward the high frequency and high reward categories.

\subsubsection{Methods}

Experiments $1 \& 2$ were completed in the Spring and Summer of 2013 at the University of Colorado, Boulder.

\section{Participants}

The first 76 participants were undergraduate students who participated to fulfill a course requirement. Subsequent participants were paid for their participation. All participants were randomly divided among the two experiments and two conditions within each experiment and worked at their own pace throughout the experiment.

\section{Stimuli}

Patterns of shapes were created as instances of either Category A or Category B. In Category A, the left outer shape matches the middle inner shape and the middle outer shape matches the right inner shape. In Category B, the left inner shape matches the middle outer shape and the middle inner shape matches the right outer shape (see Figure 1.7).

\section{Procedure}

The experiments were administered via computer in one session lasting less than one hour. Participants were divided into four groups, defined by the factorial combination of Experiment 1 versus Experiment 2 and two conditions within each experiment. In Experiment 1, the condition determined which category was high reward and which category was low reward during training. In Experiment 2, the condition determined which category was high frequency and which category was low frequency during training. In both experiments, the cover story involved training 

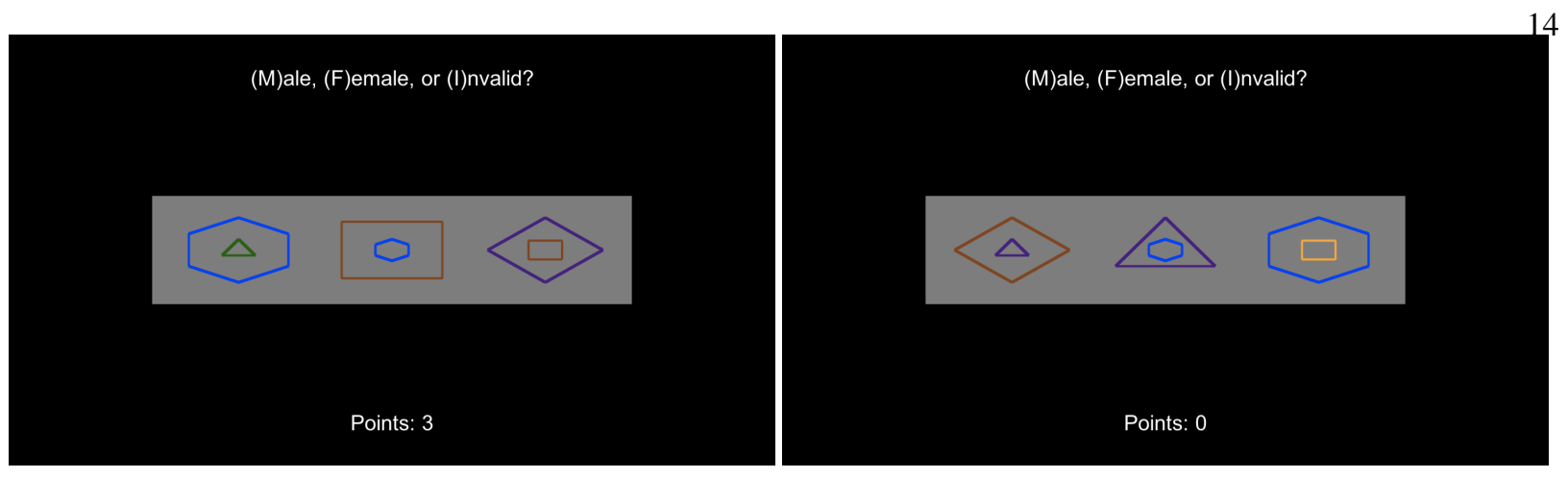

Figure 1.7: Training Trial Stimuli Examples. Left: Category A stimulus presented during a training trial. Right: Category B stimulus presented during a training trial.

participants to categorize alien nametags as male or female, and then having participants create alien nametags that were partially completed.

During the training phase, participants classified instances of each category as either "Male", "Female", or “Invalid" for 200 trials. Male and Female label assignments to categories was counterbalanced across participants. In each trial, a category instance was presented and participants responded using the "M", "F", or "I" keys. Following each response, a feedback screen with the words "Correct" or "Incorrect", the correct category label, and points earned was presented for $1.5 \mathrm{~s}$. In 10 percent of trials, the pattern of shapes did not satisfy either category definition and the correct response was “Invalid". In Experiment 1, the reward for one category (integer points sampled uniformly from the range $[4,6])$ was higher than for the other category (range $[2,4])$ and the frequencies were equal $(45 \%$ vs. $45 \%)$. In Experiment 2, the frequency of instantiation of one category was higher than the other category and the rewards were equal (both categories were worth 4 points). In both experiments, points were only given for correct responses and correct invalid responses were worth 1 point.

After training, participants were tested with a category completion task. In each trial, a partial instance of Category A or Category B was presented by replacing the rightmost pair of shapes with a question mark. In 80 percent of trials, the partial instance was unambiguous in that it could only be correctly completed as one or the other category. In the critical 20 percent of trials, the partial instance could be correctly completed as either category (see Figure 1.8). Four answer choices were presented that included the correct answer(s) and 3 foils (for the unambiguous trials) or 2 foils (for the ambiguous trials). Participants selected one of the answer choices using keys 1-4 and then saw a blank screen for 700ms with the words "Thank You". No points were given during the test task. 

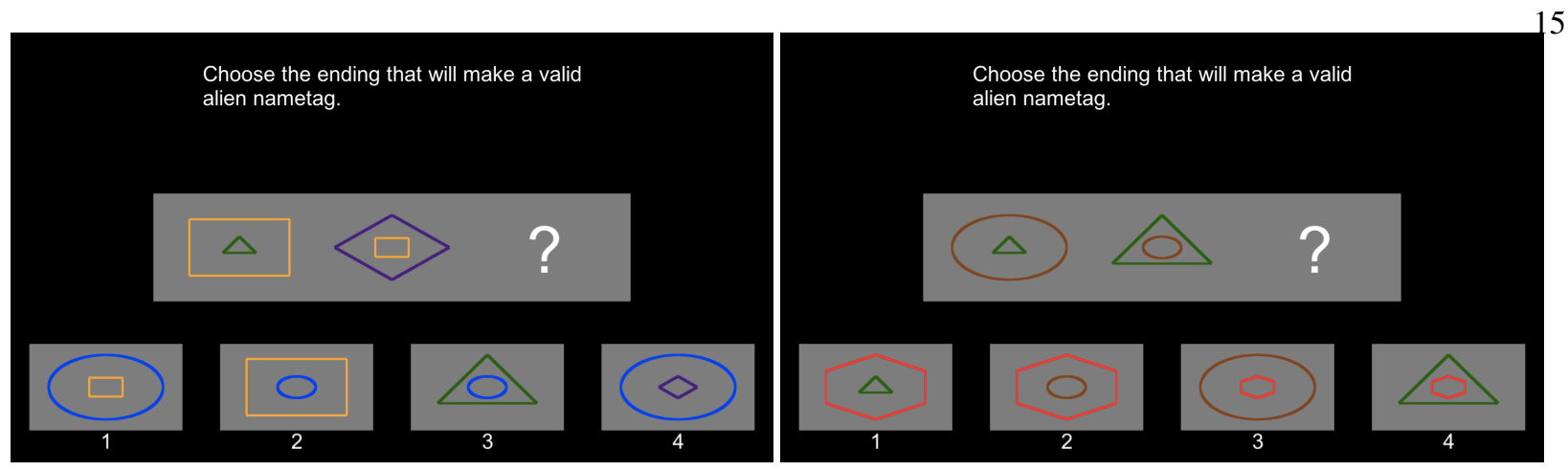

Figure 1.8: Test Trial Stimuli Examples. Left: Unambiguous Test Trial. Right: Ambiguous Test Trial.

\subsubsection{Results}

We predicted that category completion of ambiguous items will be biased toward the high frequency and high reward categories. As reported in Figure 1.9, this result was marginally significant for the frequency manipulation, but not significant for the reward manipulation.

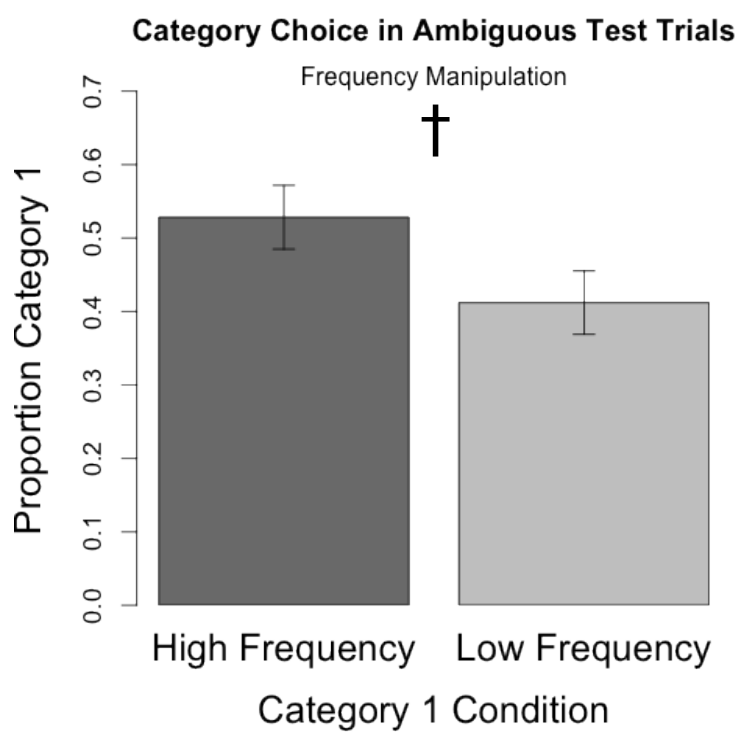

Category completion of ambiguous test items was marginally biased toward the high frequency category. $F(1,62)=3.59, p=.06$

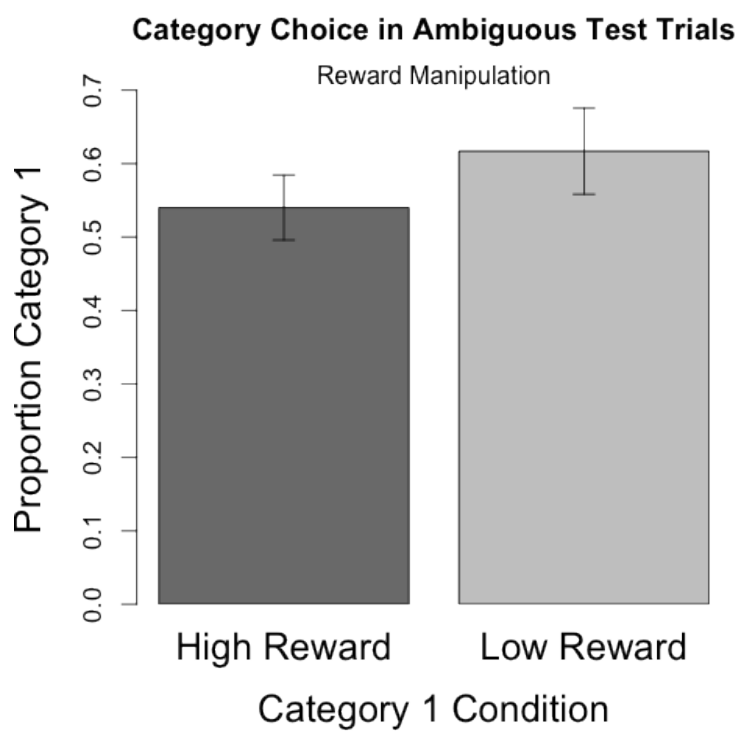

Category completion of ambiguous test items was not biased toward the high reward category. $F(1,52)=1.09, p=.30$

Figure 1.9: Shapes Results. 


\subsubsection{Experiments 1 \& 2 Discussion}

In Experiment 1, correct categorization of instances of one category was worth more points than the other category during training. This reward manipulation had a significant effect on subsequent usage of the relevant schema. However, interpretation of these results is limited because participants did not seem to process the difference in category points. The lack of effect of the reward manipulation suggests it is an open question what reward factors influence the reinforcement of relational concepts. Experiment 2 manipulated the frequency of instantiation of each category during training. Each category schema was used to complete ambiguous test items more often when it was higher frequency than when it was lower frequency. This result suggests that higher frequency does bias the retrieval and application of schemas, perhaps by increasing their strength or baseline activation.

\subsubsection{Experiment 3}

In Experiment 1, the reward mean was manipulated to create a high mean reward category and a low mean reward category. This mean manipulation did not appear to have an effect (possibly because participants did not attend to the point rewards at all). Experiment 3 manipulated the reward variance, and paid participants based on their accumulated reward points. The manipulation of reward variance made one category more predictive of actual reward outcome than the other category. In generalizing reward prediction to compound cue stimuli (stimuli which instantiate schemas from both categories), the participants' reward predictions should be biased toward the more predictive category. This prediction is supported by simulation of a simple schema strength model.

Two parts of the model were being tested in this experiment. The first test is whether participants learn differential rewards for the relational concepts (schemas). The ability to associate reward values with categories that are defined by relational properties is a basic premise of the model. One might expect this prediction not to hold because no individual stimulus is ever repeated. Above-chance performance in the test phase would suggest that participants have learned to associate values with abstract categories (schemas) and generalize those values to new instances via structural alignment between the schema and instance. The second test is whether participants learn differential strengths for the relational concepts. The computational model learns which concepts are more useful (i.e., more predictive of reward) and weights those concepts with higher strength when estimating the value of novel stimuli. In the model, lower-variance concepts (i.e., when that concept is present in the stimulus actual reward outcome varies less) acquire 


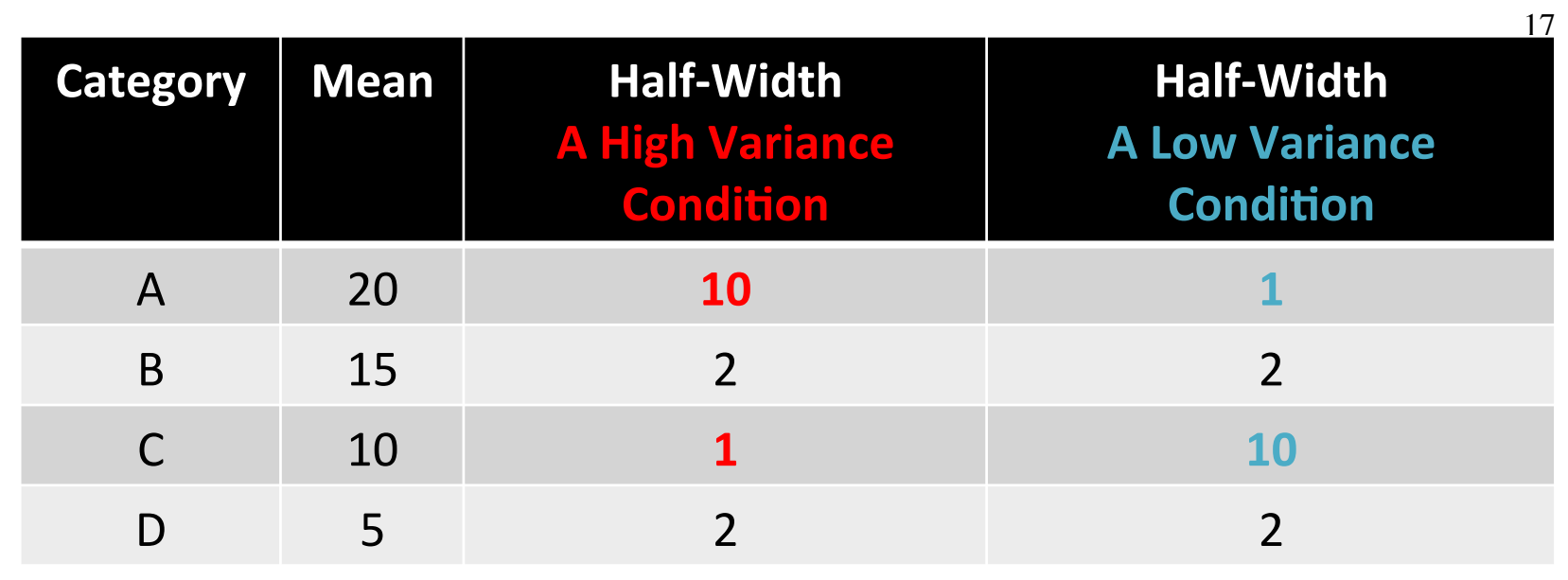

Table 1.1: Experiment 3 Conditions and Point Values. In the "A High Variance" condition (3rd column), category A had a half-width of 10 and category $\mathrm{C}$ had a half-width of 1 . In the "A Low Variance" condition (4th column), category A had a half-width of 1 and category $C$ had a half-width of 10 . The means and half-widths of categories B and D were the same across conditions. Points were sampled from triangular distributions with these means and half-widths, and then were multiplied by a scaling factor of 1.7 .

higher strength, but differences in reward mean have no effect on strength. Participants' valuation of the combined cue stimulus (AC in Figure 1.10) is a direct test of this model prediction. Participants' valuation of the combined cue stimulus AC should be biased towards the lower-variance (more predictive) category.

\section{Participants}

160 participants were drawn from Amazon Mechanical Turk.

\section{Design}

The experiment consisted of a two-alternative forced-choice task (2AFC), which was intended to make participants attend to the point means and variances (in contrast to Experiments $1 \& 2$ where participants may have only attended to whether they were correct or incorrect). Four categories of items were used in the experiment: A, B, and C, and D. Items from each category were worth a certain number of points. The variance (high vs. low) of points for Categories A \& C was manipulated in a between-subjects design. The two conditions are shown in Table 1.1. The positioning of stimuli in the 2AFC task (left vs. right side of the display) and ordering of trials was randomized. The appropriate differences in category means (high vs. low) and variances (high vs. low), and the mean for the B category were determined via a pilot study. The mean for category B was set to a "middle" mean value in between the high and low mean values so that choices for critical AC vs. B trials were not at ceiling or floor for either item.

\section{Stimuli}


Stimuli for the experiment consisted of $4 \times 4$ arrays of colored shapes (see Figure 1.10). Each $4 \times 4$ array is a single instance of one or more of the categories $(\mathrm{A}, \mathrm{B}, \mathrm{C}$, or $\mathrm{D})$. The categories are intended to be defined by self-evident patterns or properties. The simplicity of the patterns was chosen to test the effects of reward mean and variance manipulations on post-induction processes (i.e., reinforcement of the schema after it has been acquired by the participant), rather than pre-induction processes (i.e., the processes that lead participants to initially discover the category-defining schema). Property 1 is defined by objects within the same column being the same color. Property 2 is defined by objects within the same quadrant being the same color and shape. Property 3 is defined by objects within the same row being the same shape. Property 4 is defined by objects within the center, on the edges, and on the corners having unique color and shape. The AC category is defined by the presence of both properties 1 and 3 .

Items for each category were generated randomly within the constraints of the category-defining property. Importantly, the physical stimuli were counterbalanced so that for half of participants, the high-mean property was 1 , and for half of subjects, the high-mean property was 3. For the purposes of this paper, "A" refers to the high-mean category and "C" refers to the low-mean category, collapsing across the counterbalanced physical stimuli. Category B was always assigned to property 2 , and category D was always assigned to property 4 .

\section{Procedure}

The cover story for the experiment framed the task as diving for oysters. Participants were told they must select between two oysters on each trial, and that the pattern on an oyster's shell reflects the value (size) of the pearl inside. This cover story was designed so that participants would interpret both the shell pattern and the pearl value as caused by some latent variable of the oyster. This causal structure was intended to get participants to value the combined cue stimulus (AC) somewhere in between the values of A and C (i.e., use an averaging model where the valuation of AC would be some weighted average of the values of A and C). If instead participants expected the value of AC stimuli to be greater than either $\mathrm{A}$ or $\mathrm{C}$ (i.e., additive model where the valuation of $\mathrm{AC}$ would be the sum of the values of $\mathrm{A}$ and C), then they would likely always choose AC over B and the experimental predictions would not hold.

The procedure for the experiment involved a training phase followed by a testing phase. The training phase involved repeated trials of a selection task in which participants chose between two items on each trial (two alternative forced choice, 2AFC). Participants were instructed to choose the oyster that they predicted would be worth more points. After each choice, feedback was given in the form of points for only the chosen item. Participants had to learn which 


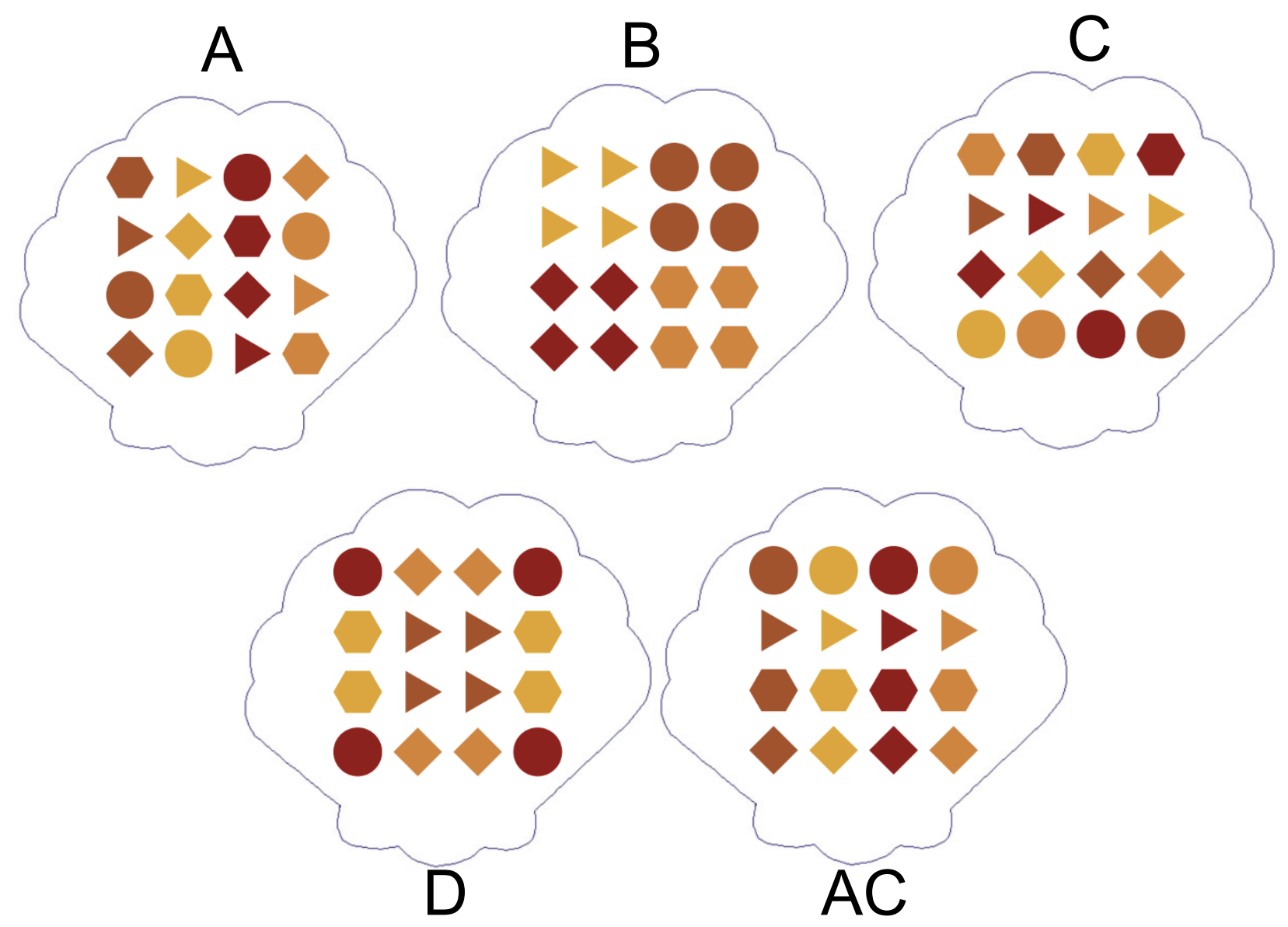

Figure 1.10: Stimuli Examples for Experiment 3. 
categories of items were associated with higher reward. They had to learn these value associations from feedback on the number of points their selection was worth. Giving feedback on only the chosen item required participants to learn the means and variances of all the categories in order to know whether they were "correct" or not (i.e, whether they had chosen the higher-valued item).

Training trials consisted of 3 trial types, where participants were presented choices between categories A vs. B, B vs. C, or C vs. D. After sufficient training (determined by reaching criterion of 5 consecutive correct choices for each trial type, or a maximum of 100 training trials), participants began the test phase. The test phase was the same as the training phase, but also included 10 critical AC vs. B trial types in addition to 10 trials from each of the training trial types. These AC compound cue items instantiated schemas from both categories A and C. No feedback was given on the test trials.

\subsubsection{Predictions}

The main hypothesis for this experiment was that predictions about novel situation outcomes should be biased towards sources of relational knowledge that have been more reliably predictive. In other words, participants should rely more on highly predictive concepts and less on less predictive concepts when generalizing to situations that instantiate both concepts. Specifically, we predicted that: 1. Participants would learn to choose stimuli with the

higher-valued properties. 2. Valuation of combined cue AC stimuli would be biased towards the less variable property (see Figure 1.11). When the high mean property was highly variable, participants would tend to choose B over AC. When the low mean property was highly variable, participants would tend to choose AC over B.

\subsubsection{Results}

Only participants who performed well during non-critical test trials were included in these analyses, leaving 90 participants out of 160 total. There were 30 non-critical test trials (10 each of A v. B, B vs. C, and C vs D), and 10 critical test trials (AC v. B). Inclusion criterion for participants was 20 or more correct non-critical test trials. This criterion was set because it corresponded to a natural break in the histogram of participant performance, and also corresponds to the number of correct trials a participant would need to be significantly above chance performance.

The main result aligns with our predictions. Participants chose AC over B more often in the "A is low variance" 


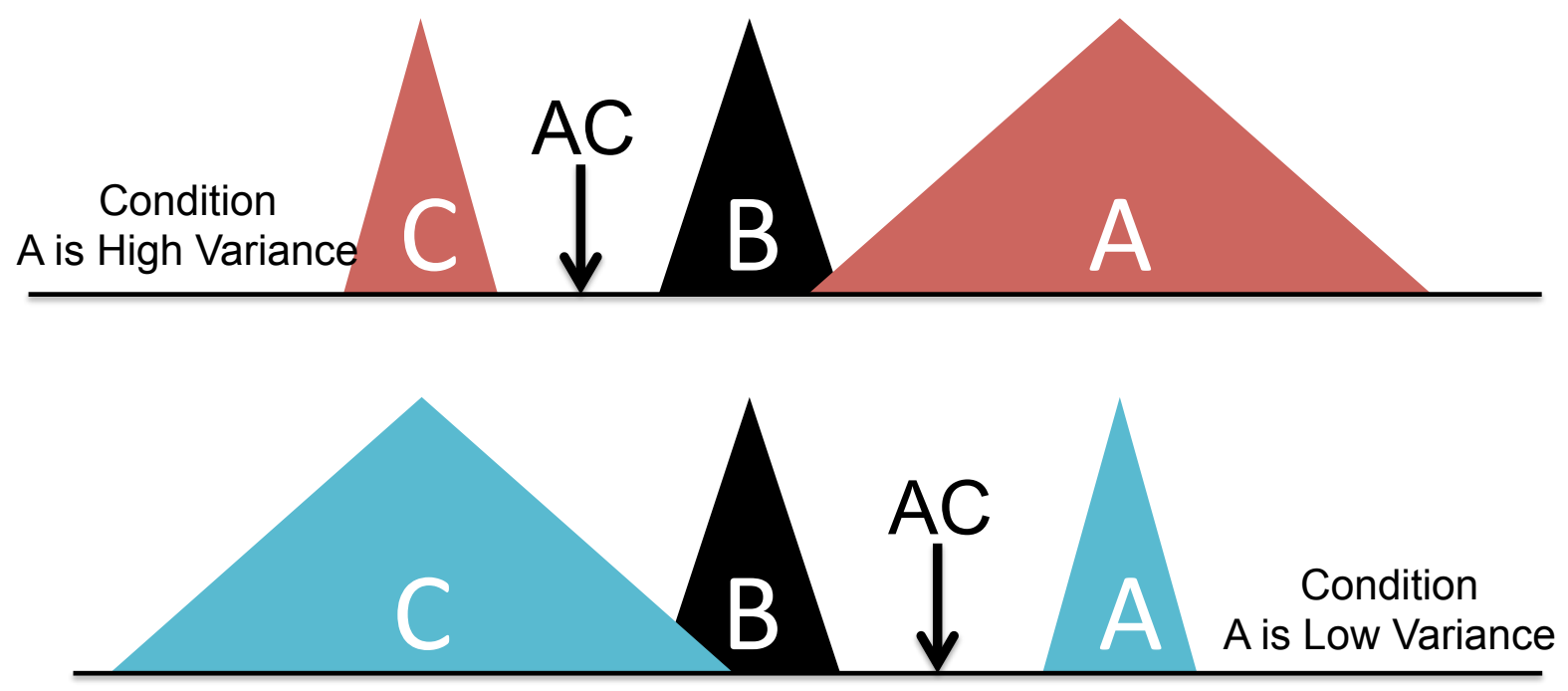

Figure 1.11: Predictions for Experiment 3. The horizontal lines represent abstract number lines and the A, B, and C triangles represent the means and variances of their respective triangular distributions. We predicted that the valuation of the $\mathrm{AC}$ items would be shifted from the arithmetic mean of $\mathrm{A}$ and $\mathrm{C}$ (which was equal to the mean of $\mathrm{B}$ ) towards the lower-variance category. In the "A is High Variance" condition (top, in red), it was predicted that the valuation of AC items would be shifted towards C, resulting in participants selecting B over AC in critical test trials. In the "A is Low Variance" condition, it was predicted that the valuation of $\mathrm{AC}$ items would be shifted towards A, resulting in participants selecting AC over B in critical test trials. 
condition than in the "A is high variance" condition (see Figure 1.12).

The next two results serve as manipulation checks, showing that participants were sensitive to the variance manipulation. Figure 1.13 shows that training accuracy was better in trials involving the lower-variance category. Figure 1.14shows that test response time was faster in trial types involving the lower-variance category.

\subsubsection{Experiment 3 Discussion}

As predicted, participants were able to learn the category structures and associate the categories with quantitative values based on feedback. They learned to select the higher mean category during training even though the specific stimuli on each trial hadn't been seen before. Participants were sensitive to reward mean and variance in both training and testing phases. They performed more accurately and responded faster to trials involving the lower-variance category.

During testing, as predicted, AC was chosen more when A was the low variance category. In other words, participants appeared to weight the lower-variance category more in estimating the value of the combined cue stimulus. This result supports the hypothesis that people rely more on concepts that have been more predictive of reward when faced with an ambiguous situation.

Future work will replicate the experiment with participants in the laboratory. We expect that the current results should also replicate with simple featural categories. Connections to traditional associational learning models are considered in the general discussion.

This experiment provides evidence for a schema strength mechanism qualitatively similar to the one specified in the computational model. We hypothesize that stronger schemas should be weighted higher in predicting the value of new situations, recognized faster, and be more likely to retrieved from memory. Further evidence for these hypotheses

could come from a similar experiment that uses single-item category verification time as a measure of schema strength. Stronger schemas should be verified faster when participants are presented with the combined-cue stimulus.

This experimental paradigm could also be extended to cases of richer schema usage, where the participant's action is related to how the schema pattern is instantiated in the stimulus. In these richer cases, the schema might affect participants' causal interpretation of the stimulus. 


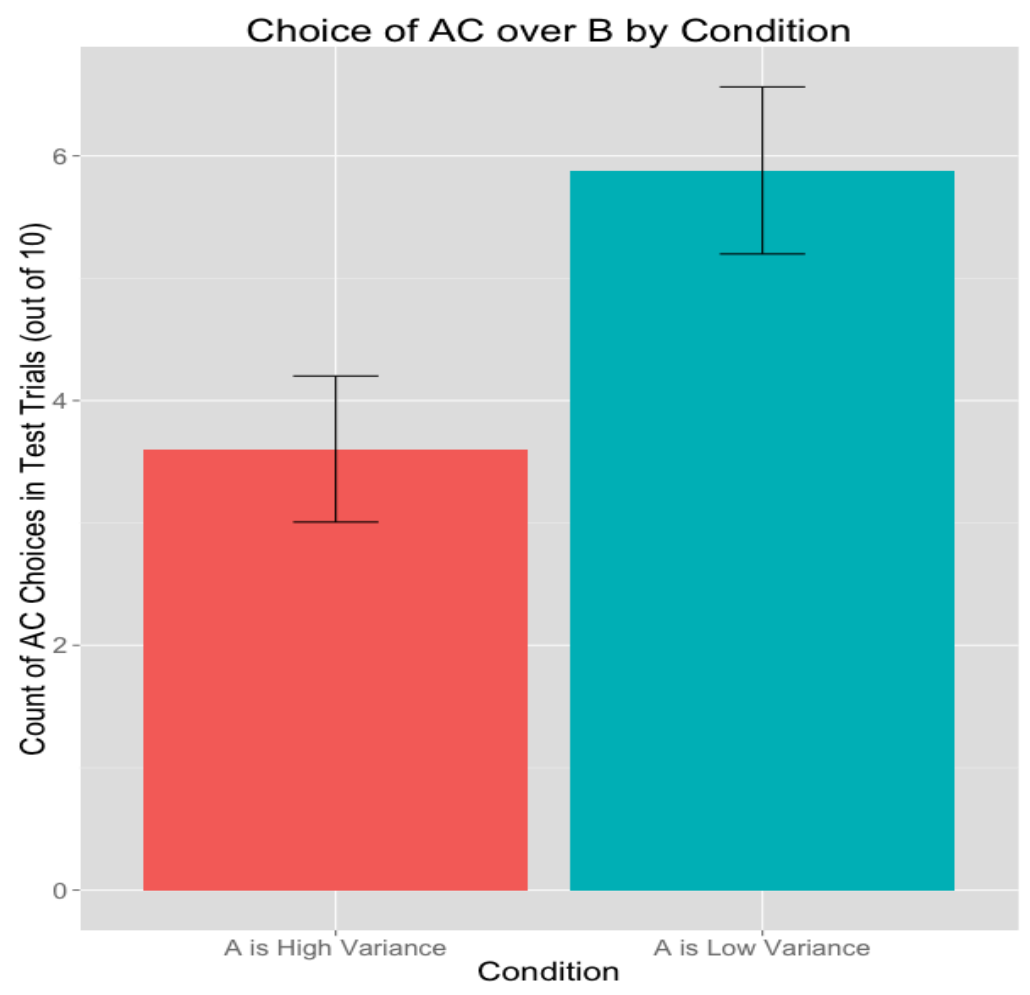

Figure 1.12: Critical Test Trials. Condition significantly predicts choice of AC in AC vs. B trials in the expected direction (difference in means $=2.28, p<.05$ by both t-test and Mann-Whitney)
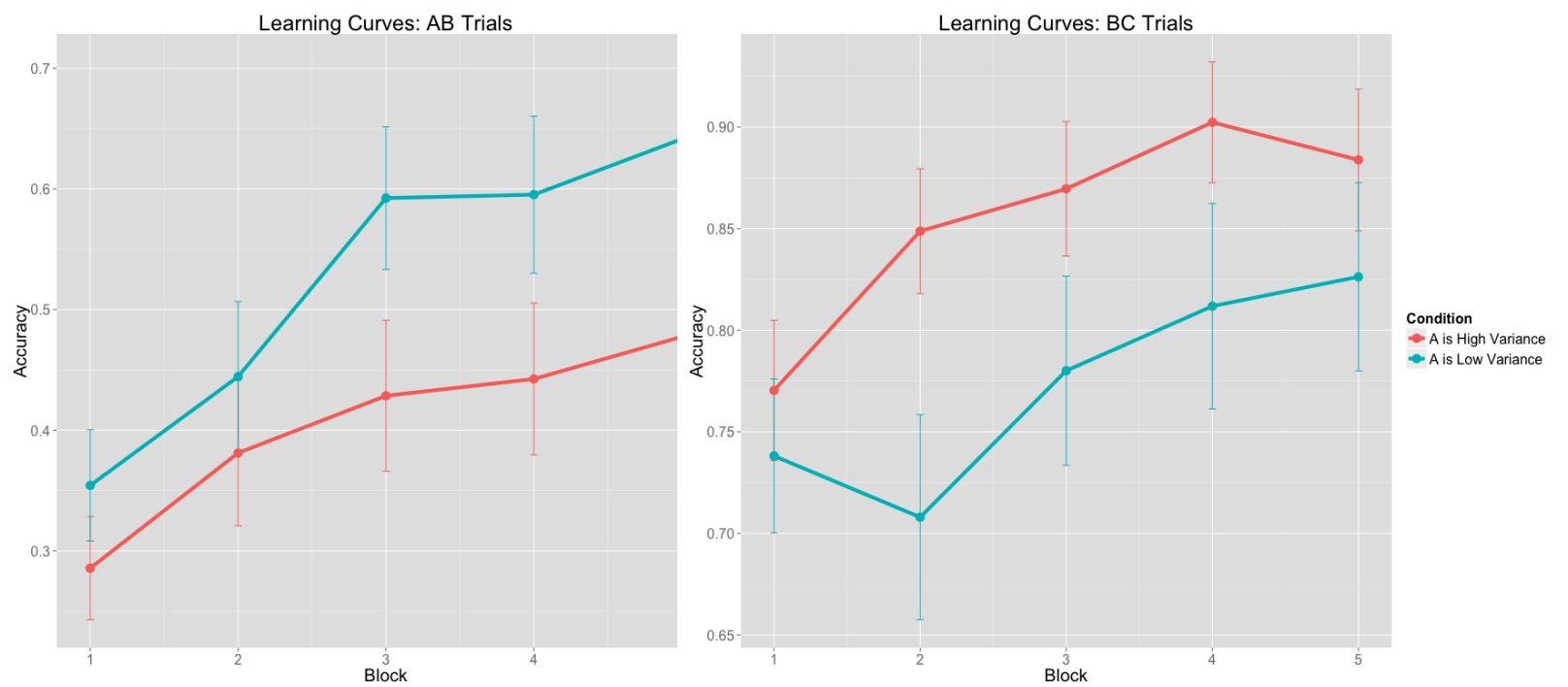

Figure 1.13: Training Trial Accuracy. There was a significant interaction between Trial Type and Condition in accuracy on training trials $(t(78)=-2.07, p<.05$ by mixed model analysis of variance). Accuracy is better on AB trials when $\mathrm{A}$ is low variance than when $\mathrm{A}$ is high variance (left). Accuracy is better on $\mathrm{BC}$ trials when $\mathrm{C}$ is low variance than when $\mathrm{C}$ is high variance (right). For this figure, blocks were defined by averaging over sequences of 20 trials. Participants who reached criterion were assigned an accuracy of 1 for trials they were not presented. 


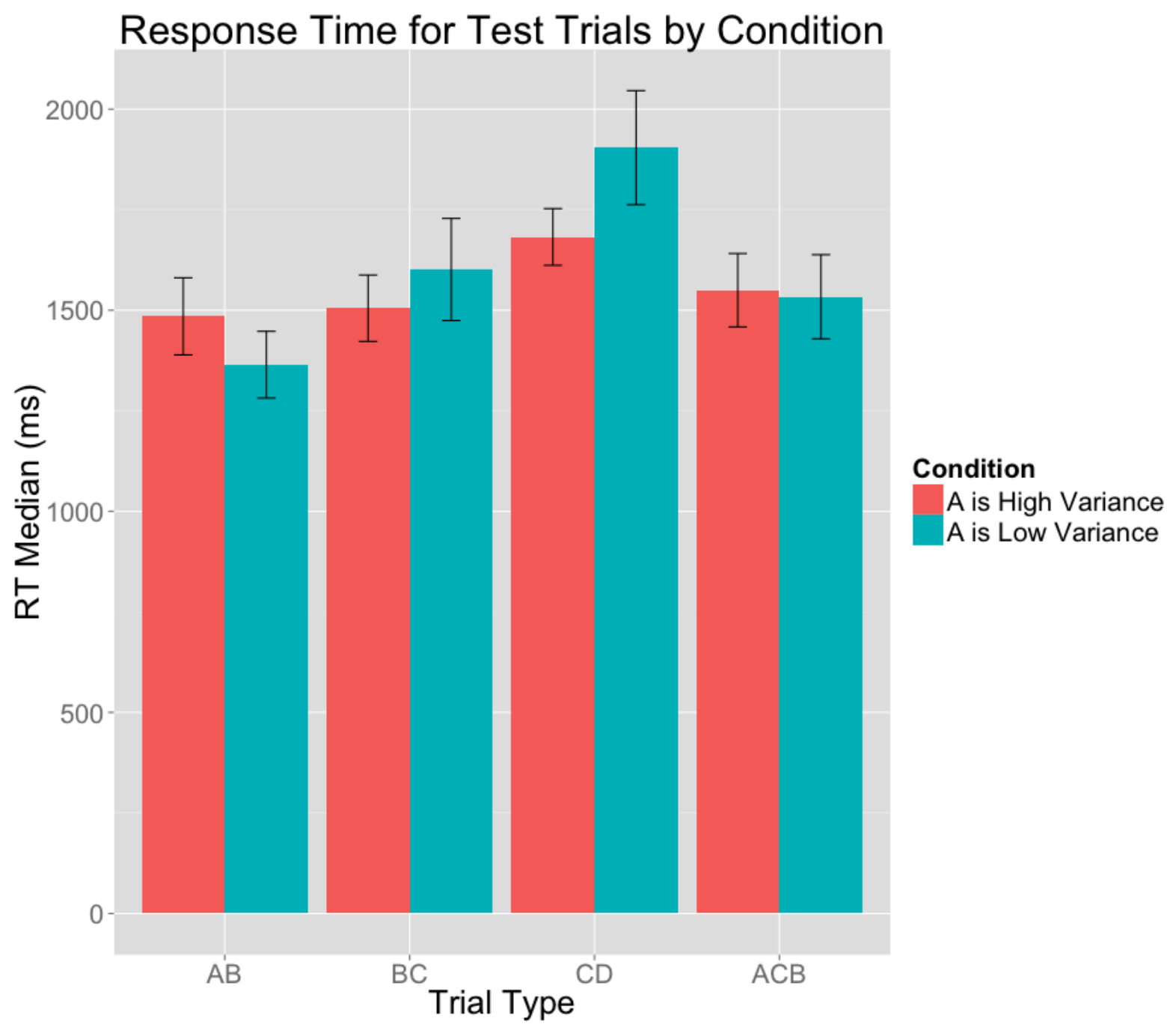

Figure 1.14: Test Trial Response Times. There was a significant interaction between Trial Type (AB v. BC) and Condition on response time $(t(88)=2.00, p<.05)$. Response time is faster on $\mathrm{AB}$ trials when $\mathrm{A}$ is low variance than when $\mathrm{A}$ is high variance, and faster on $\mathrm{BC}$ trials when $\mathrm{C}$ is low variance than when $\mathrm{C}$ is high variance. 


\subsection{General Discussion}

The results presented here constitute a proof-of-principle that analogy and schema induction can be productively integrated with a learning framework founded on RL and similarity-based generalization. This integration leads to a model exhibiting sophisticated, abstract generalization derived from analogical similarity, as well as discovery of new higher-order relational concepts driven by their ability to predict reward.

The basic modeling framework used here applies not just to analogical similarity and schema induction, but to other forms of representational learning as well. Kernel-based RL offers a powerful and general theory of representation learning, because it can be integrated with any form of representation that yields a pairwise similarity function. Its TD error signal can drive changes in representation via the objective of improving generalization. This idea has been applied to learning of selective attention among continuous stimulus dimensions (Jones and Cañas, 2010). The current model offers a richer form of representation learning, in that it acquires new concepts rather than reweighting existing features.

The analogical RL model also builds on other models of relational learning. Tomlinson and Love (2006) propose a model of analogical category learning, with essentially the same similarity and exemplar generalization mechanisms adopted in the present model. Our model adds to theirs in that it applies to dynamic tasks and in that it grows its representation through schema induction. Van Otterlo (2012) has developed methods for applying RL to relational representations of the same sort used here, although the approach to learning is quite different. His models are not psychologically motivated and hence learn in batches and form massive conjunctive rules, with elaborate updating schemes to keep track of the possible combinations of predicates. In contrast, the present approach learns iteratively, behaves probabilistically, and grows its representation more gradually and conservatively. This approach is likely to provide a better account of human learning, but a more interesting question may be whether it offers any performance advantages from a pure machine-learning perspective.

In the present model, the activation of each exemplar elicited by a candidate state can be thought of as a feature of that state. The exemplar effectively has a "receptive field" within the state space, defined by the similarity function. This duality between exemplar- and feature-based representations is founded in the kernel framework (see Shawe-Taylor and Cristianini, 2004). The present model takes advantage of this duality, producing a smooth transition 
from an episodic, similarity-based representation to a more semantic, feature-based representation defined by learned schemas.

The value and attention learning mechanisms in the model that are tested in the experiments have roots in traditional associational learning models. The classic Rescorla-Wagner model introduced joint learning of cue-outcome associations, but had no mechanism for attention learning (Rescorla et al., 1972). Although the Rescorla-Wagner model allowed for different input cues to have different associabilities, there was no mechanism for learning these attentions. Mackintosh (1975) introduced an attention learning mechanism which modifies attention to cues to reduce prediction error and reduce interference between cues. Support for this attention learning mechanism comes from demonstrations of learned inattention in experiments with rats (Mackintosh and Turner, 1971) and humans (Kruschke and Blair, 2000).

The analogical RL attention-learning mechanism in the present work is compatible with Mackintosh's theory and with extensions by Kruschke (2001). Each of these models would predict that attention would increase to lowervariance cues (as found empirically in Experiment 3 above), based on the idea that attention increases to cues that are more predictive (contribute less error), compared to either the average individual cue predictiveness (as in Mackintosh (1975) and Kruschke (2001)'s mixture of experts model) or compared to the overall combined cue predictiveness (as in Kruschke (2001)'s EXIT model and the present ARL model).

The present work is complementary to hierarchical Bayesian models that discover relational structure through probabilistic inference (Tenenbaum et al., 2011). Whereas our model builds up schemas from simpler representations, the Bayesian approach takes a top-down approach, defining the complete space of possibilities a priori and then selecting among them. The top-down approach applies to any learning model, because any well-defined algorithm can always be circumscribed in terms of its set of reachable knowledge states. This is a useful exercise for identifying inductive biases and absolute limits of learning, but it offers little insight into the constructive processes that actually produce the learning. The present model offers proposals about the mechanisms underlying how the human mind discovers new, abstract concepts.

\subsection{Future Directions}

The following ideas represent future directions of this work. 


\subsubsection{The Role of Language in Relational Concept Learning}

Of primary interest is establishing the conceptual mapping between concept/category learning and language in order to bridge analogical reinforcement learning with language processing. Establishing this mapping involves translating the representations of relational role-binding, structure-mapping, and schema induction to the language domain, as well as importing theories and models of language processing into the analogical reinforcement learning framework. Such a mapping should facilitate the design of experiments to test the framework experimentally and to extend the model. Two potential interactions between ARL and language are (1) the effect of words on comparison, schema induction, and consolidation, and (2) learning syntax, semantic roles, and subcategorization frames using ARL.

\subsubsection{The Role of Words in Comparison, Schema Induction, and Consolidation}

Language is almost certainly an important factor in learning higher-order relations, and may also facilitate relational consolidation. Relational consolidation is the process by which systems of relations (schemas) transition into unitary objects which can be recognized perceptually (rather than by structure-mapping), retrieved from memory in parallel, and participate in yet higher-order relations (Foster et al., 2012). Son et al. (2010) offer two possible roles of language in relational learning: "(1) words invite learners to compare, highlight, and represent relations (the Generic Tokens [GT] hypothesis), and/or (2) words carry semantic cues to common structure (the Cues to Specific Meaning [CSM] hypothesis)" (p. 55). Thompson et al.'s (1997) finding that chimpanzees' learning higher-order relations depended on initial training with material tokens can be interpreted as support for the GT hypothesis. In humans, words can act as linguistic tokens (Clark, 2006) and have been shown to aid category learning (Lupyan et al., 2007). The importance of words as generic tokens seems highly compatible with our view of the power of atomizing (i.e., tokenizing) higher-order relations.

In other words, the presence of a label could trigger comparison with other things that have the same label. The label might also help guide schema induction by tagging concepts that are useful to learn, as well as aiding recognition of instances of the concept. And labels could facilitate consolidation by providing a unitary token to serve as a stand-in and common perceptual element for the complete, complex concept.

Experiments testing the role of words in these processes would compare the learning of higher order concepts 
with and without useful labels. In the computational model, words could be introduced as tokens which represent larger systems of relations. The model could be extended to include a mechanism of consolidation that depends on the presence of the word tokens.

\subsubsection{Learning Semantic Roles and Subcategorization Frames}

It is possible that the ARL framework could be used as a model of language learning. Octavian Popescu's ICS Colloquium on "Sense Discrimination Patterns and Verb Clustering" (2013) outlined a computational approach to identifying sense discrimination patterns (SDPs) in language. These SDPs can be used as templates for natural language processing tasks such as word sense disambiguation (identifying which meaning of a word that has multiple meanings is meant in context), textual entailment, machine translation, and verb clustering. For example, an SDP for one sense of the word 'abandon' is: [human | institution] abandon [activity | plan]. The SDP encodes a specification of the ordering and semantic types of the verb's arguments. The pattern can be used to identify instances of this sense of 'abandon' in a corpus that has been properly pre-processed. In addition, SDPs can be learned from example sentences. Popescu used a finite state automata learning algorithm (Angluin) to infer a set of SDPs from example sentences in a human-made NLP resource. Importantly, SDPs can be viewed as patterns of role-binding structure. The verb is a relation which has semantic roles which are bound to noun-phrase arguments. In the above example, abandon is a relation that has two semantic roles: [human | institution] and [activity | plan]. These semantic roles can be bound to instances of the specified type, such as "The United States of America" and "the troop surge in Iraq", respectively. The meaning of a sentence such as "The United States of America abandoned the troop surge in Iraq" is represented in part by the verb and the order and type of its roles. The notion that sentence meaning can be represented by relational role-binding structure suggests that theories and machinery of relational representation processing can be applied to language processing. Therefore, it may be possible to learn SDPs by processes of analogy and schema induction. In such a framework, SDPs could be inferred by comparing paired examples of sentences with the same verb, searching for the best mapping between them, and inducing a schema that captures their shared structure.

In psycholinguistics, the ARL model parallels theories about how abstract word classes are learned as subcategorization frames. Subcategorization frames specify the syntactic position of the verb's arguments, the part of speech of each argument, and the semantic features required of each argument. Infants may initially learn and store 
these structures separately for each verb, but eventually develop abstract subcategorization frames that can represent the structure for multiple similar verbs. These abstractions may be responsible for the sudden explosive growth of vocabulary in infants. Simulations with the ARL model could test whether the learning and performance of the model replicates the vocabulary explosion phenomenon.

However, it is not clear what the error signal would be in the acquisition of language. One possibility is that people make on-line predictions about the next word in a sentence, and errors in that prediction are used to update linguistic representations. Further exploration is needed to formalize the connections between ARL and language. 


\section{Bibliography}

Clark, A. (2006). Language, embodiment, and the cognitive niche. Trends in Cognitive Sciences, 10(8):370-4.

Doumas, L. A. A., Hummel, J. E., and Sandhofer, C. M. (2008). A theory of the discovery and predication of relational concepts. Psychological Review, 115:1-43.

Forbus, K., Gentner, D., and Law, K. (1995). MAC/FAC: A model of similarity-based retrieval. Cognitive Science, 19(2):141-205.

Forbus, K. D. and Gentner, D. (1989). Structural evaluation of analogies: What counts. Proceedings of the 11th Annual Conference of the Cognitive Science Society, pages 341-348.

Foster, J. M., Cañas, F., and Jones, M. (2012). Learning conceptual hierarchies by iterated relational consolidation. Proceedings of the 34th Annual Conference of the Cognitive Science Society, pages 324-329.

Foster, J. M. and Jones, M. (2013). Analogical reinforcement learning. Proceedings of the 35th Annual Conference of the Cognitive Science Society.

Gentner, D. (1983). Structure-mapping: A theoretical framework for analogy. Cognitive Science, 7(2):155-170.

Gick, M. L. and Holyoak, K. J. (1980). Analogical problem solving. Cognitive Psychol, 12:306-355.

Hummel, J. E. and Holyoak, K. J. (2003). A symbolic-connectionist theory of relational inference and generalization. Psychological Review, 110:220-264.

Jones, M. and Cañas, F. (2010). Integrating reinforcement learning with models of representation learning. Proceedings of the 32nd Annual Conference of the Cognitive Science Society, pages 1258-1263.

Kruschke, J. K. (2001). Toward a unified model of attention in associative learning. Journal of Mathematical Psychology, 45(6):812-863.

Kruschke, J. K. and Blair, N. J. (2000). Blocking and backward blocking involve learned inattention. Psychonomic Bulletin \& Review, 7(4):636-645.

Lupyan, G., Rakison, D. H., and McClelland, J. L. (2007). Language is not just for talking: Redundant labels facilitate learning of novel categories. Psychological Science, 18(12):1077-83.

Mackintosh, N. and Turner, C. (1971). Blocking as a function of novelty of cs and predictability of ucs. The Quarterly Journal of Experimental Psychology, 23(4):359-366.

Mackintosh, N. J. (1975). A theory of attention: Variations in the associability of stimuli with reinforcement. Psychological Review, 82(4):276.

Markman, A. B. and Gentner, D. (1993). Structural alignment during similarity comparisons. Cognitive Psychol, 25:431-431. 
Rescorla, R. A., Wagner, A. R., et al. (1972). A theory of pavlovian conditioning: Variations in the effectiveness of reinforcement and nonreinforcement. Classical conditioning II: Current research and theory, 2:64-99.

Rummery, G. A. and Niranjan, M. (1994). On-line q-learning using connectionist systems. Technical Report CUED/FINFENG/TR 166, Cambridge University.

Shawe-Taylor, J. and Cristianini, N. (2004). Kernel Methods for Pattern Analysis. Cambridge University Press.

Son, J. Y., Doumas, L. A. A., and Goldstone, R. L. (2010). When do words promote analogical transfer? Journal of Problem Solving, 3(1):52-92.

Sutton, R. S. and Barto, A. G. (1998). Reinforcement Learning: An Introduction. The MIT Press.

Tenenbaum, J. B., Kemp, C., Griffiths, T. L., and Goodman, N. D. (2011). How to grow a mind: Statistics, structure, and abstraction. Science, 331:1279-1285.

Thompson, R. K., Oden, D. L., and Boysen, S. T. (1997). Language-naive chimpanzees (Pan troglodytes) judge relations between relations in a conceptual matching-to-sample task. Journal of Experimental Psychology. Animal Behavior Processes, 23(1):31-43.

Tomlinson, M. T. and Love, B. C. (2006). From pigeons to humans: Grounding relational learning in concrete examples. Proceedings of the 21st National Conference on Artificial Intelligence (AAAI-06), pages 199-204.

Van Otterlo, M. (2012). Solving relational and first-order logical markov decision processes: A survey. Reinforcement Learning, 12:253-292. 